\title{
Caso clínico: adulto con anomalía de Ebstein
}

\author{
Clinical case: adult with Ebstein anomaly \\ Pacheco Rodríguez Jennifer Paola', Torres Washima Irene \\ Lucía², Serrano Barbecho Wladimir Augusto $^{3}$
}

VOLUMEN 38 | N² 2 | AGOSTO 2020

FECHA DE RECEPCIÓN: 20/02/2020 FECHA PUBLICACIÓN: 31/08/2020 FECHA DE REVISIÓN: 18/01/2021 FECHA DE REEDICIÓN: 15/03/2021

1. Hospital de especialidades José Carrasco Arteaga. Cirugía. Hemodinámica. Bulán - Ecuador

2. Hospital de especialidades José Carrasco Arteaga. Cirugía Cirugía cardiotorácica. Cuenca - Ecuador

3. Universidad de Cuenca. Facultad de Ciencias Médicas. Docente de la carrera de Medicina. Cuenca - Ecuador

\begin{tabular}{c|c} 
Caso & $\begin{array}{c}\text { Clinical } \\
\text { Case }\end{array}$ \\
\hline
\end{tabular}

DOI: https://doi.org/10.18537/RFCM.38.02.05

Correspondencia:

jennpao.pachecorodriguez@gmail.com

Dirección:

La pirámide. Bulán

Código Postal:

010102

Teléfono:

072341387 - 0995003396

Bulán - Ecuador

\section{RESUMEN}

Introducción: la anomalía de Ebstein se caracteriza por la implantación baja de las valvas septal y posterior de la tricúspide; por lo tanto, la aurícula derecha es amplia y su ventrículo es pequeño. Al ser una patología bastante rara en nuestro medio se encuentra subdiagnosticada y no recibe el tratamiento oportuno. Por ende, la importancia de conocerla para no retrasar su manejo clínico quirúrgico.

Caso clínico: paciente de 46 años de edad, masculino, con antecedente de hipoacusia congénita, presentó disnea (clase funcional III) más palpitaciones y dolor torácico atípico hace 1 año aproximadamente. Al examen físico evidenció soplo holosistólico en foco tricuspídeo y uso moderado de musculatura accesoria. Al ecocardiograma transesofágico, se observó arritmia auricular más implante bajo de las valvas de la tricúspide y se inició tratamiento clínico más ablación del flutter. En la evolución presentó mejoría de la sintomatología y dependiendo del deterioro de la clase funcional, tratamiento quirúrgico.

Conclusiones: en el presente caso se ajustó tratamiento clínico y ablación del flutter de acuerdo a sintomatología del paciente, mostrando mejoría.

Palabras clave: anomalía de Ebstein, aleteo atrial, cardiopatías congénitas. 


\section{ABSTRACT \\ Introduction: Ebstein's anomaly is characterized by low implantation of the septal and posterior tricuspid leaflets; therefore, the right atrium is wide and its ventricle is small. Being a rather rare pathology in our environment, it is underdiagnosed and does not receive the appropriate treatment. Therefore, it is important to know it to avoid a delay in its clinical surgical management.}

Clinical case: The patient is 46-year-old male, with a history of congenital hearing loss, who presented dyspnea (functional class III) plus palpitations and atypical chest pain approximately 1 year ago. In the physical examination, he revealed a holosystolic murmur in the tricuspid focus and moderate use of accessory muscles. A transesophageal echocardiogram revealed atrial arrhythmia plus implantation of the tricuspid leaflets and it was started a clinical treatment plus flutter ablation. In the evolution he presented improvement of the symptoms and depending on the deterioration of the functional class, surgical treatment.

Conclusions: in this case, clinical treatment and flutter ablation were adjusted according to the patient's symptoms, showing improvement.

Key words: Ebstein anomaly, atrial flutter, congenital heart disease.

\section{INTRODUCCIÓN}

La anomalía de Ebstein se trata de una cardiopatía congénita con desplazamiento apical del anillo tricuspídeo siendo su incidencia de 1 por cada 20000 recién nacidos vivos [1]. La particularidad de ésta patología es que las valvas de la tricúspide no se unen normalmente y se encuentran desplazadas hacia abajo en la cavidad ventricular derecha
[2]. Además, la misma se relaciona con una comunicación interauricular en un $70 \%$ de los casos y continúa en frecuencia la estenosis pulmonar y la atresia pulmonar [3]. También, el $25 \%$ de los casos se asocia a vías de conducción accesorias [4]. En recién nacidos sintomáticos, menos del 50\% sobreviven a los 5 años y aquellos diagnosticados en la adultez tienen buen pronóstico pos quirúrgico [1,4].

La malformación de Ebstein está definida por: a) condición de la válvula tricúspide; b) compromiso funcional del ventrículo derecho (VD), dependiente de la "porción auriculizada", y c) ritmo auricular [3]. Por lo tanto, la ecocardiografía determina un diagnóstico preciso para decisiones terapéuticas y pronóstico en la enfermedad de Ebstein [5]. En ésta generalmente se visualiza un desplazamiento apical de las valvas tricúspides: septal y posterior, que superan los $8 \mathrm{~mm}$ con relación a la implantación de la válvula mitral en el adulto [6].

Actualmente, la ecocardiografía y la resonancia magnética (RM) cardíaca son claves para calcular el momento y el tipo de cirugía. La RM brinda una excelente cuantificación del tamaño y la función del ventrículo derecho [7]. En tanto, que el cateterismo cardíaco se lo realiza cuando se considere cirugía [8].

Las recomendaciones para cirugía son: deterioro de clase funcional según la escala de New York Heart Association (NYHA), cianosis, cardiomegalia y dilatación progresiva del ventrículo derecho [4]; sin embargo, se sugiere una intervención quirúrgica antes del desarrollo de enfermedad severa [7]. La ablación con catéter en arritmias auriculares en pacientes con enfermedad de Ebstein tendría un resultado favorable con un perfil de recurrencia y seguridad aceptable [9]. En cambio, la ablación quirúrgica del flutter auricular es efectiva en 
pacientes que serán sometidos a cirugía por anomalía de Ebstein y que no son susceptibles de tratamiento percutáneo $[10,4]$.

\section{REPORTE DEL CASO}

Paciente masculino de 46 años de edad, casado, de etnia mestiza, procedente y residente de Riobamba. Refiere desde edad escolar presentar disnea de grandes esfuerzos sin otra sintomatología, hace un año aproximadamente la misma se exacerba a medianos esfuerzos acompañada de palpitaciones más dolor torácico atípico y astenia. No refiere consumo de alcohol ni tabaco, cuenta con antecedente de hipoacusia congénita e insuficiencia tricúspide.

Hallazgos clínicos: al examen físico, tensión arterial: 130/60, saturación de oxígeno: 90\% con fracción inspirada de oxígeno: 21, frecuencia cardíaca: 90-120, frecuencia respiratoria: 22. Corazón: ruidos cardiacos arrítmicos, soplo holosistólico en foco tricuspídeo de intensidad de $3 / 6$ en escala de Levine. Campos pulmonares: uso moderado de musculatura accesoria.

Exámenes complementarios: a) Radiografía de tórax: cardiomegalia estadio III (ver Imagen $\left.N^{\circ} 1\right)$, b) Electrocardiograma: flutter auricular, conducción 3:1, c) Ecocardiograma: fracción de eyección del ventrículo izquierdo (FEVI): $62 \%$. Dilatación de aurícula derecha (AD) por implantación apical de válvula tricúspide $12 \mathrm{~mm}$ con relación al implante de válvula mitral, presentó reflujo severo (ver Imagen $\mathrm{N}^{\circ} 2$ ); d) Cateterismo Cardíaco: insuficiencia tricuspídea severa. Presión sistólica de arteria pulmonar: $20 \mathrm{mmHg}$.
Imagen $\mathrm{N}^{\circ} 1$

Radiografía de tórax

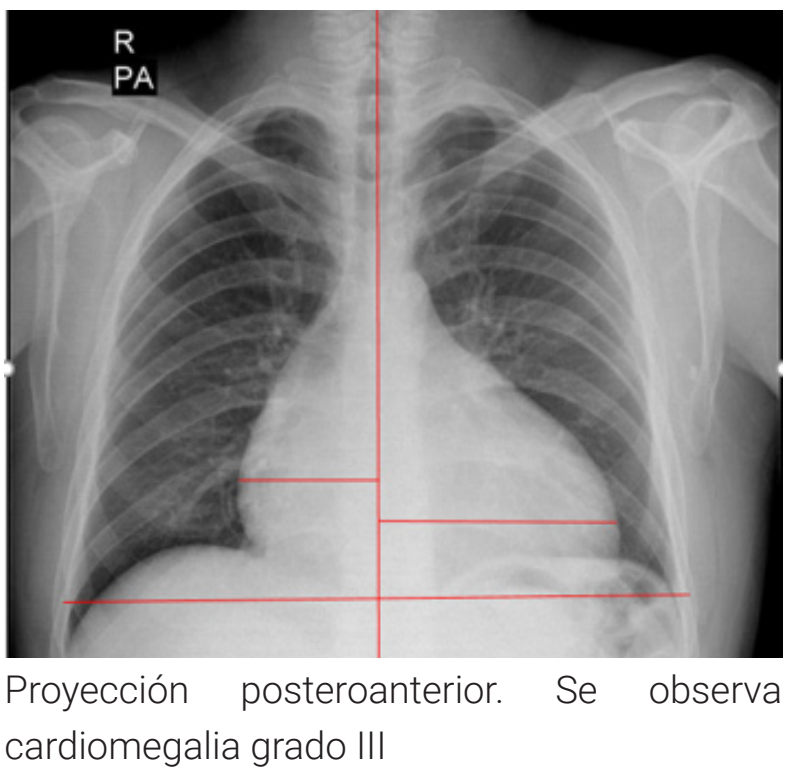

Imagen $\mathrm{N}^{\circ} 2$

Ecocardiograma tras esofágico

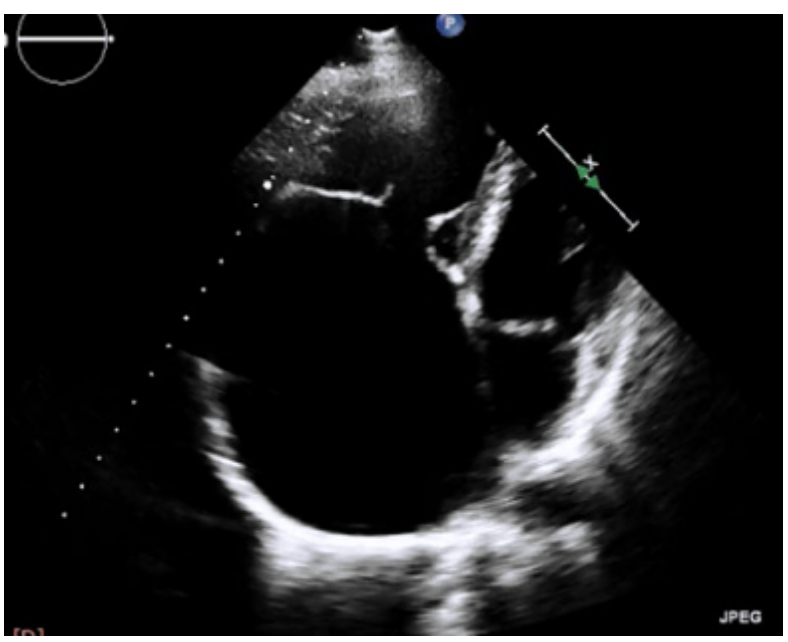

Se observa la implantación anómala baja de la válvula tricúspide en el ventrículo derecho (VD), lo cual produce un aumento del diámetro de la aurícula derecha (AD) a expensas del VD.

Esta patología al tratarse de una enfermedad congénita cardíaca de válvula tricúspide, debe realizarse el diagnóstico diferencial principalmente con la displasia y atresia de la válvula tricúspide. En éste contexto, los presentes diagnósticos diferenciales no encajan con el cuadro del paciente, ya que se trata de un adulto que hasta hace 
poco presentó una buena clase funcional. Generalmente, las patologías mencionadas muestran un cuadro de presentación precoz; en cambio, en la anomalía de Ebstein, el inicio de su presentación dependerá del grado de disfunción de la válvula tricúspide.

En los pacientes que presentan la enfermedad de forma moderada o asintomáticos, tienen en general pronóstico bueno ya que el estilo y esperanza de vida es normal. Lo cual coincide con el presente caso pues se trata de una variante moderada (Clasificación de Carpentier B) en cuanto a la presentación clínica porque fue hasta hace poco que desarrolló ésta sintomatología.

La intervención terapéutica incluyó amiodarona 200mg VO BID, carvedilol 6.25mg VO BID, furosemida 40mg VO QD, espironolactona 25mg VO QD y posteriormente se realizó ablación de flutter auricular sin complicaciones, cuya conclusión diagnostica fue en territorio supra ventricular, presencia de flutter auricular, ablación de flutter auricular. Conducción auricular ventricular alterada y territorio ventricular con estabilidad eléctrica ventricular con estabilidad eléctrica ventricular.

Seguimiento: en el presente caso las manifestaciones clínicas (disnea de medianos esfuerzos, palpitaciones acompañado de dolor torácico atípico y astenia) se debió al flutter auricular; por lo tanto, realizará ablación con catéter, valorar evolución clínica y dependiente de ésta se planteará a tratamiento quirúrgico, basándonos en el deterioro de la clase funcional como indicación para plastia o cambio de la válvula tricúspide. Como problemas coexistentes durante la evaluación al paciente, hubo dificultad en la comunicación debido a la hipoacusia congénita que presentaba; por lo mismo, se realizó la evaluación con ayuda de un traductor de señas.

\section{DISCUSIÓN}

La anomalía de Ebstein es de baja incidencia y por lo tanto rara [1]. Existe un porcentaje pequeño de pacientes que son diagnosticados en edad adulta por complicaciones. Hay una alta morbilidad de ésta enfermedad en edades tempranas. Por lo tanto, es primordial un diagnóstico precoz para un tratamiento oportuno, ya que al ser una patología subdiagnosticada y no recibir la atención necesaria presentará un peor pronóstico.

A ésta patología se la asocia a trastornos de la conducción cardíaca y a la existencia de CIA, en la mayoría de los casos en un 25\% [4]. Asimismo, su forma de presentación con inserción baja de las valvas de la tricúspide produce disfunción de la misma y causará insuficiencia cardíaca a expensas de las cámaras derechas. La ecocardiografía transtorácica, transesofágico y actualmente RM cardíaca son las técnicas de imagen primordiales para determinar la enfermedad de forma precisa.

En estos pacientes es importante individualizar cada caso con sus respectivas enfermedades concomitantes o antecedentes, para así definir entre tratamiento clínico, electrofisiológico, hemodinámico y/o quirúrgico. Por otra parte, según la literatura se sugiere que la cirugía sea temprana, antes que empeore el cuadro clínico [7]. Sin embargo, se debería considerar un tratamiento integral clínico- quirúrgico para el bienestar del paciente; por lo tanto, dependiendo del caso habrá una respuesta favorable al ajuste terapéutico, ablación por catéter, cierre percutáneo del CIA y/o tratamiento quirúrgico.

Es así que, la ablación con catéter en arritmias auriculares como en el caso de nuestro paciente tiene un resultado favorable con un perfil de recurrencia y seguridad aceptables [9]. No así, la ablación quirúrgica del flutter 
auricular es efectiva en pacientes que serán sometidos a cirugía por no ser susceptibles a tratamiento percutáneo $[10,4]$. Cabe indicar que en sí el cateterismo cardiaco en pacientes con enfermedad de Ebstein denota un riesgo por el progreso de arritmias desencadenando consecuencias graves [11] y aún más en el caso expuesto por el antecedente.

Entonces al combinar tratamiento clínico y ablación por catéter, la clase funcional del paciente mejoró (CF II), las palpitaciones desaparecieron y el dolor torácico atípico es infrecuente. Se corroboró que la mencionada sintomatología era secundaria al flutter auricular y no directamente a su patología de base; así mismo, se encuentra categorizado el caso según la clasificación de Carpentier en tipo B [12]. No obstante, es necesario realizar el seguimiento del paciente y en caso de deterioro funcional, se optará por el tratamiento quirúrgico. Conjuntamente comparando con otro tipo de alteraciones congénitas cabe indicar que la técnica ablación con catéter puede resultar complicada por los defectos anatómicos y las alteraciones del sistema de conducción. También siendo importante mencionar que por este tipo de situaciones la ablación por catéter puede incluso resultar fallida y necesitar de ablación quirúrgica [13]. Por lo tanto, es fundamental realizar el respectivo seguimiento al caso estudiado.

Además; se han elaborado otros tipos de estudios, donde la ablación con catéter de las arritmias producidas por la malformación de Ebstein constituyen un tratamiento significativo y a su vez logra establecer la indicación primaria de tratamiento quirúrgico [14].

\section{CONCLUSIÓN}

La anomalía congénita de Ebstein es una enfermedad valvular subdiagnosticada, con manifestaciones cardíacas que deterioran la capacidad funcional y en muchos casos no recibe el tratamiento, siendo importante reconocerla oportunamente.

\section{PERSPECTIVA DEL PACIENTE}

Refiere mejoría en cuanto a su sintomatología con tratamiento combinado efectuado.

\section{ABREVIATURAS}

CIA: Comunicación inter auricular.

FEVI: fracción de eyección del ventrículo izquierdo.

NYHA: New York Heart Association.

VD: Ventrículo derecho.

VI: Ventrículo izquierdo.

AD: Atrio derecho.

VO: Vía oral.

QD: 1 vez por día.

BID: 2 veces por día.

\section{ASPECTOS BIOÉTICOS}

El presente estudio se efectuó bajo total confidencialidad de datos personales del paciente y con el respectivo consentimiento informado.

\section{INFORMACIÓN DE LOS AUTORES}

Pacheco Rodríguez Jennifer Paola. Médica. Hospital de Especialidades José Carrasco Arteaga. Cirugía. Hemodinámica. Bulán-AzuayEcuador.

e-mail:jennpao.pachecorodriguez@gmail.com ORCID:https://orcid.org/0000-0002-9917-7943

Torres Washima Irene Lucía. Médica. Hospital de especialidades José Carrasco Arteaga. Cirugía Cardiotorácica. Cuenca - Azuay Ecuador.

e-mail: ire_12_17@hotmail.com

ORCID:https://orcid.org/0000-0002-2330-2509 
Serrano Barbecho Wladimir Augusto. Doctor en Medicina y Cirugía. Especialista en Traumatología y Ortopedia. Universidad de Cuenca. Facultad de Ciencias Médicas. Carrera de Medicina.

e-mail:wlacho20@yahoo.com

ORCID:https://orcid.org/0000-0003-2339-3786

\section{CONTRIBUCIÓN DE LOS AUTORES}

Todos los autores contribuyeron de manera similar en la concepción y diseño del trabajo, búsqueda bibliográfica y análisis crítico del documento.

\section{CONFLICTO DE INTERESES}

Los autores declaran no tener conflictos de intereses.

\section{FUENTES DE FINANCIAMIENTO}

Autofinanciado

\section{REFERENCIAS BIBLIOGRÁFICAS}

1. Elsevier. Anomalía de Ebstein: la cardiopatía congénita y (casi) anónima [Internet]. Elsevier Connect. [citado 9 de febrero de 2020]. Disponible en: https://www.elsevier. com/es-es/connect/medicina/anomaliade-ebstein-la cardiopatia-congenita-y-casianonima.

2. Frescura, C. Angelini, A. Daliento, L. Thiene, G. Morphological Aspects of Ebstein's Anomaly in Adults. Thorac cardiovasc Surg. Rev. PubMed. 2000 Aug;48(4):203-8. DOI: 10.1055/s-2000-6893.

3. Patiño, E. Yabur, M. Muñoz, L. Calderón, J. Buendía, A. Anomalía de Ebstein y Comunicación Interventricular. Una asociación poco frecuente. Arch. Cardiol. Méx. 2009 Mar;79(1):41-5. URL:http:// www.scielo.org.mx/scielo.php? script $=$ sci_
arttext\&pid=S140599402009000100008 \&lng=es\&tlng=es

4. Warnes, C. Williams, R. Bashore, T. Child, J. Connolly, H. Dearani, J. Guidelines for the Management of Adults with Congenital Heart Disease. Journal of the American College of Cardiology. ACC/AHA. 2008 Dec;52(23):143263. URL: https://doi.org/10.1161/ CIRCULATIONAHA.108.190811

5. Muñoz, L. Espinola, N. Kuri, M. Keirns, C. Ebstein's Anomaly: Anatomoechocardiographic correlation, Cardiovascular Ultrasound. BMC. 2007 Nov; 5(43): 1-10. DOI: 10.1186/1476-71205-43.

6. Oechslin, E. Buchholz, S. Jenni, R. Ebstein's Anomaly in Adults: DopplerEchocardiographic Evaluation. Rev. PubMed. 2000 Aug;48(4):209-13. DOI: 10.1055/s-2000-6900.

7. Qureshi, M. O'Leary, P. Connolly, H. Cardiac imaging in Ebstein anomaly. Trends in Cardiovascular Medicine. Rev. PubMed. 2018 Jan;28(6):403-9. DOI: 10.1016/j. tcm.2018.01.002.

8. Baumgartner, A. Falk, V. Bax, J. Bonis, M. Hamm, C. Holm, P. Guía ESC/EACTS 2017 sobre el tratamiento de las valvulopatías. Rev Esp Cardiol. 2018 Feb;71(2):110-47. DOI: 10.1016/j.recesp.2017.12.014.

9. Hassan, A. Tan, N. Aung, H. Connolly, H. Hodge, D. Vargas, E. Outcomes of atrial arrhythmia radiofrequency catheter ablation in patients with Ebstein's anomaly. Rev. PubMed. 2018 Mar;20(3):535-40. DOI: 10.1093/europace/euw396.

10. Stulak, J. Sharma, V. Cannon, B. Ammash, 
N. Schaff, H. Dearani, J. Optimal surgical ablation of atrial tachyarrhythmias during correction of Ebstein Anomaly. Rev. PubMed. 2015 May;99(5):1700-1705. DOI: 10.1016/j.athoracsur.2015.01.037.

11. Attie, F. Calderon, J. Zabal, C. Buendia, A. Enfermedad de Ebstein. Cardiología Pediátrica. 2da edición. Estados Unidos. Editorial Médica Panamericana; 2012. 149 p. ISBN 9786077743743.

12. Cruz, E. Ivy, D. Jaggers, J. Cardiac Surgery and Intensive Care. Pediatric and Congenital Cardiology. 1era edición. United States. Springer-Verlag London; 2014. ISBN 978-3-030-62294-7.

13. Benito, F. Sánchez, C. Torres, V. Ablación con catéter de vías accesorias en pacientes con cardiopatías congénitas. Rev Esp Cardiol. 1999; 52(1): 1028-1031. URL: https://www. revespcardiol.org/day 06/01/2021

14. Ramírez, S. Cervantes, J. Anomalía de Ebstein. Arch. Cardiol. Méx. 2006; 76(2):6568. URL: www.archcardiolmex.org.mx 